\title{
Synthesis and Characterization of Nanoporous Hydroxyapatite with High Hemocompatibility
}

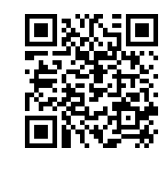

\author{
Chee-Heong Ooi, Swee-Yong Pung and Fei-Yee Yeoh* \\ School of Materials and Mineral Resources Engineering, Universiti Sains Malaysia, Malaysia
}

Received: June 05, 2018; Published: June 18, 2018

*Corresponding author: Fei-Yee Yeoh, School of Materials and Mineral Resources Engineering, Engineering Campus, Universiti Sains Malaysia, 14300 Nibong Tebal, Penang, Malaysia

\begin{abstract}
Hydroxyapatite with nanoporous structure is a valuable biomaterial to enhance the efficiency of adsorption based biomedical applications, i.e., drug delivery and protein adsorption. In this study, nanoporous hydroxyapatite was synthesized by using hydrothermal technique. Decyltrimethylammonium bromide (C10TAB) surfactant was used as template for the formation of nanoporous structure in the hydroxyapatite. The presence of pure hydroxyapatite phase in the synthesized samples is verified by Fourier transform infrared spectroscopy. The nanoporous hydroxyapatite samples exhibit pore size of $18.5-33.5 \mathrm{~nm}$, BET surface area of $39.5-84.6 \mathrm{~m}^{2} \mathrm{~g}^{-1}$ and pore volume of $0.35-0.44 \mathrm{~cm}^{3} \mathrm{~g}^{-1}$. The hemolysis test shows that the nanoporous hydroxyapatite samples are highly hemocompatible with the percentage of hemolysis less than 5 $\%$. This study reveals the effectiveness of the hydrothermal technique for producing nanoporous hydroxyapatite targeted for adsorption based biomedical applications.
\end{abstract}

Keywords: Hydroxyapatite; Porous Material; Calcination; Surface Area; Hemolysis

Abbreviations: BET: Brunauer Emmett Teller; BJH: Barrett Joyner Halenda

\section{Introduction}

Hydroxyapatite is a useful biomaterial with a composition similar to that of the human bone and has an excellent biocompatibility, bioactivity as well as non-immunogenicity [1]. However, commercial hydroxyapatite exhibits very low porosity and it cannot be used effectively for adsorption based biomedical applications, especially drug delivery, protein and nucleic acid fractionation [2]. Hydroxyapatite with high porosity is a valuable biomaterial for adsorption-based biomedical applications as it promotes better adsorption capacity and kinetics. Hydrothermal technique involving the use of surfactant is a preferable method to synthesize hydroxyapatite with desired porosity $[3,4]$. The addition of surfactant provides template for hydroxyapatite crystal growth. The surfactant is then removed via washing and calcination process. Calcination is a heat treatment process used to prepare hydroxyapatite with high purity and uniform particle size, thus it is commonly applied in hydroxyapatite synthesis process.Hemocompatibility is a vital study to be performed prior to the utilization of hydroxyapatite in blood related biomedical applications. When blood is in contact with non-hemocompatible material, it causes the activation of coagulation and immune systems in human body which may threaten a patient's life [5]. Therefore, it is necessary to conduct hemocompatibility study especially hemolysis test on the nanoporous hydroxyapatite to ensure the safety of patients.In this study, nanoporous hydroxyapatite was synthesized based on a surfactant-templating system using decyltrimethylammonium bromide (C10TAB). The effect of calcination on the physicochemical properties and hemocompatibility of the synthesized hydroxyapatite is studied.

\section{Materials and Methods}

\section{Synthesis of Hydroxyapatite}

Hydroxyapatite was synthesized by using hydrothermal technique reported previously [6]. $100 \mathrm{~mL}$ of phosphate solution $(0.24 \mathrm{M})$ was prepared by dissolving $3.74 \mathrm{~g} \mathrm{NaH}_{2} \mathrm{PO}_{4} \cdot 2 \mathrm{H}_{2} \mathrm{O}$ (Merck) and $3.36 \mathrm{~g}$ decyltrimethylammonium bromide (C10TAB, Tokyo Chemical Industry) into deionized water. The phosphate solution was added slowly into $0.67 \mathrm{M}$ of calcium solution $\left(\mathrm{CaCl}_{2} \cdot 2 \mathrm{H}_{2} \mathrm{O}\right.$ added into $60 \mathrm{~mL}$ deionized water) until white slurry formed and the mixture was aged at $120{ }^{\circ} \mathrm{C}$ for 24 hours. The $\mathrm{pH}$ of the solutions was kept at 11 using $\mathrm{NaOH}$ solution (1M, Merck). The obtained white precipitate was washed with deionized water and dried overnight in an oven. The cleaned sample was calcined in a furnace at $550^{\circ} \mathrm{C}$ for $6 \mathrm{~h}$ to burn off the surfactant (Sample A). Similar procedure was repeated to synthesize hydroxyapatite without going through calcination process (Sample B). 


\section{Characterization}

FTIR spectrometer (Perkin-Elmer Spectrum One) was used to determine the functional groups present on the hydroxyapatite samples using $\mathrm{KBr}$ technique and scanned from the range of 4000 to $400 \mathrm{~cm}^{-1}$. Micromeritics ASAP 2020 automated gas sorption analyzer was used to determine the physisorption of the hydroxyapatite towards nitrogen $\left(\mathrm{N}_{2}\right)$ gas at $-196^{\circ} \mathrm{C}$. The surface area of the hydroxyapatite was determined from the nitrogen adsorption isotherm by using Brunauer-Emmett-Teller (BET) and Langmuir models. Barrett-Joyner-Halenda (BJH) theory was used to calculate the pore volume and pore width of the hydroxyapatite.

\section{Hemocompatibility Study}

Hemolysis test was carried out based on the experiment reported [7]. Fresh human blood from healthy donor was collected into anticoagulated tubes (K2EDTA). Five different concentrations of samples were prepared by adding the hydroxyapatite to sterile saline solution. $0.02 \mathrm{~mL}$ of anticoagulated blood was added to the tubes containing hydroxyapatite samples, distilled water (positive control) and sterile saline solution (negative control). Then, the tubes were incubated for 60 minutes in a water bath shaker at 37 ${ }^{\circ} \mathrm{C}$. After the incubation was finished, the tubes were centrifuged at $750 \times \mathrm{g}$ for 10 minutes using Eppendorf centrifuge (model 5810R). The absorbance of the supernatant at $540 \mathrm{~nm}$ was measured using spectral scanning multimode reader (Thermo Scientific, Varioskan Flash). The percentage of hemolysis was calculated using Equation (1).

Percentage of hemolysis $(\%)=\left(D_{h}-D_{n}\right) /\left(D_{p}-D_{n}\right)(1)$

where $D_{h}, D_{n}$ and $D_{p}$ are the absorbance of the hydroxyapatite, negative and positive controls, respectively. The experiments were performed using fresh blood from three healthy donors.

\section{Results and Discussion}

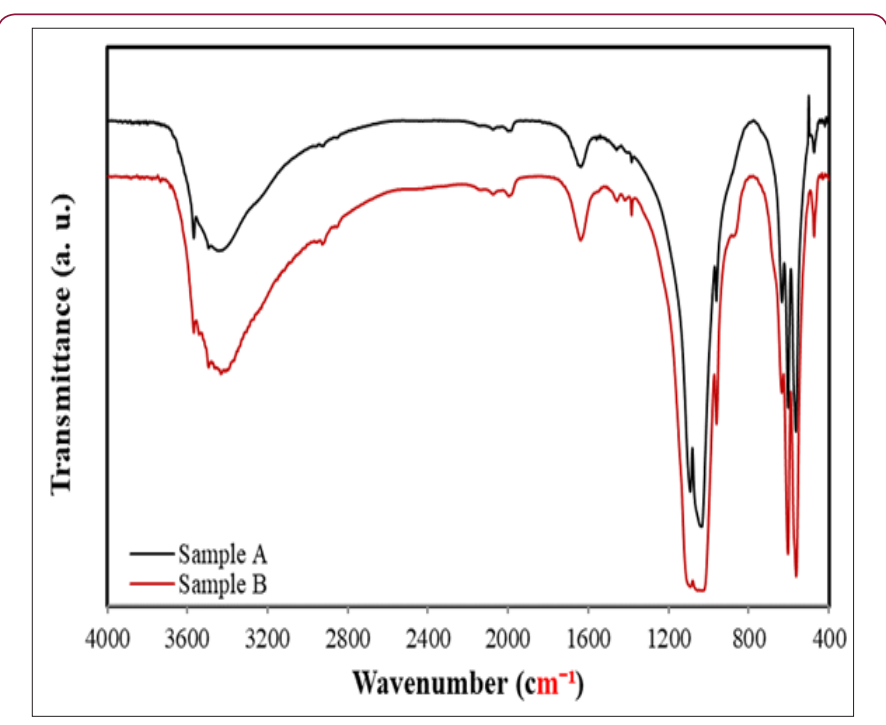

Figure 1: Fourier transform infrared spectra of the synthesized hydroxyapatite.

Figure 1 shows the FTIR spectra of the Sample A and Sample B ranged from 4000 to $400 \mathrm{~cm}^{-1}$. Sample B exhibits peaks at 3436 and
$1639 \mathrm{~cm}^{-1}$ which are attributed to the $\mathrm{H}-\mathrm{O}-\mathrm{H}$ bending of adsorbed moisture. The small peaks at $2930-2888$ and $1998 \mathrm{~cm}^{-1}$ are assigned to the alkyl groups $\left(\mathrm{CH}_{\mathrm{x}}\right)$ from $\mathrm{C} 10 \mathrm{TAB}$ carbon chains. The presence of carbonate group $\left(\mathrm{CO}_{3}^{2-}\right)$ at $1419 \mathrm{~cm}^{-1}$ is due to the adsorption of atmospheric carbon dioxide gas during synthesis process. The intensity of the $\mathrm{H}-\mathrm{O}-\mathrm{H}, \mathrm{C}-\mathrm{H}$ and $\mathrm{CO}_{3}{ }^{2-}$ peaks were relatively lower in the FTIR spectrum of Sample A. This is due to the removal of moisture, surfactant and carbon dioxide gas from the sample during calcination process. The Sample A and Sample B demonstrate an absorption band at 3570 which is associated to the stretching mode of $\mathrm{OH}$ group [8].

The characteristic peaks at 1091, 1034 and $960 \mathrm{~cm}^{-1}$ are corresponded to the P-O stretching mode of phosphate group. The bands at 604,563 and $473 \mathrm{~cm}^{-1}$ are assigned to the $0-\mathrm{P}-0$ bending mode of the phosphate group. The result shows that the Sample A and Sample B are pure hydroxyapatite without the presence of impurity. This implies that the calcination process does not cause significant difference in the chemical property of the Sample A as compared to the Sample B. The pore characteristics of the nanoporous hydroxyapatite are presented in Table 1 . The pore size for the Sample A and Sample B are 33.5 and $18.5 \mathrm{~nm}$, respectively. The pore size of the hydroxyapatite is larger than $2 \mathrm{~nm}$, suggesting the samples contain mesoporous structure. The surface area and pore volume of the Sample A are relatively lower than that of the Sample B. This is due to the effect of calcination process which has altered the porosity of the hydroxyapatite (Sample A).

Table 1: Pore characteristics of HAP and HAF samples.

\begin{tabular}{|c|c|c|c|c|}
\hline Sample & $\begin{array}{l}\text { Pore size } \\
\text { (nm) }\end{array}$ & $\begin{array}{l}\text { BET sur- } \\
\text { face area } \\
\left(\mathrm{m}^{2} \mathrm{~g}^{-1}\right)\end{array}$ & $\begin{array}{l}\text { Langmuir } \\
\text { surface } \\
\text { area }\left(\mathrm{m}^{2}\right. \\
\left.\mathrm{g}^{-1}\right)\end{array}$ & $\begin{array}{c}\text { Pore vol- } \\
\text { ume }\left(\mathrm{cm}^{3}\right. \\
\left.\mathbf{g}^{-1}\right)\end{array}$ \\
\hline Sample A & 33.5 & 39.5 & 54.6 & 0.38 \\
\hline Sample B & 18.5 & 84.6 & 123.2 & 0.54 \\
\hline
\end{tabular}

During the calcinations process, the hydroxyapatite particles coalesced to formed larger particles [9] and excess of heat has destroyed the porous structure in the sample. The result indicates that the calcination process does not improve the pore characteristics of the hydroxyapatite. Hence, a hydrothermal technique without involving calcination process is an effective way to produce nanoporous hydroxyapatite with better pore characteristics.The percentage of hemolysis of the nanoporous hydroxyapatite sample is shown in Figure 2. The increase in the hydroxyapatite concentration from 1 to $16 \mathrm{mg} \mathrm{mL}^{-1}$ do not cause significant change in the percentage of hemolysis of the samples (based on One-way ANOVA, $p>0.05, n=6)$. The percentage of hemolysis of the samples at all concentrations is ranged from 1 to $2.56 \%$. By referring to theASTM F 756-00 standard, the hydroxyapatite with percentage of hemolysis less than $5 \%$ is a highly hemocompatible and safe biomaterial [10]. There is no significant difference in the percentage of hemolysis for the Sample A and Sample B (based on paired t-test, $\mathrm{p}>0.05$, $\mathrm{n}=6$ ). This shows that the Sample A and Sample B exhibit similar hemocompatibility, suggesting that calcination does not affect the hemocompatibility of the samples. 


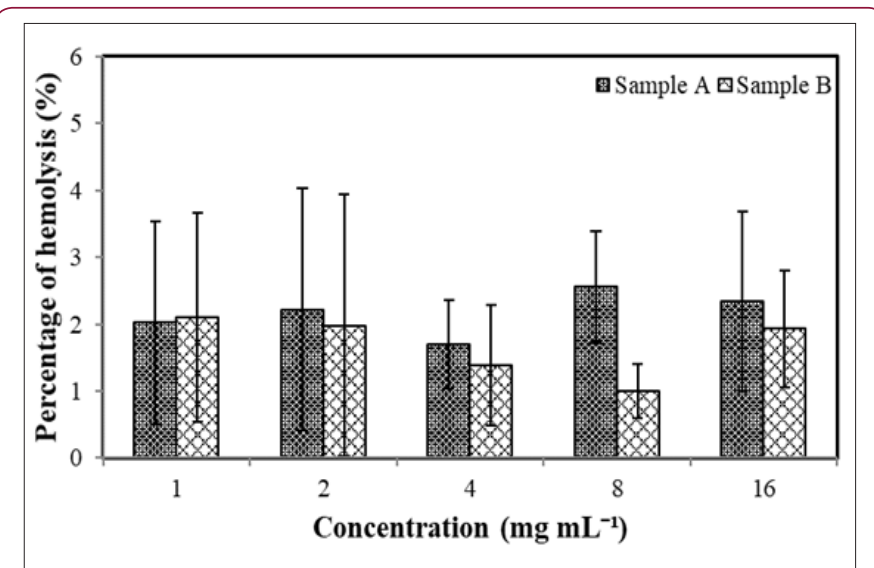

Figure 2: Percentage of hemolysis for the synthesized hydroxyapatite samples.

\section{Conclusion}

Nanoporous hydroxyapatite was successfully prepared by using hydrothermal technique and C10TAB surfactant was used as template to create porous structure in the sample. FTIR result indicates that the synthesized samples are pure hydroxyapatite. The hydroxyapatite prepared without calcination process (Sample B) shows better pore characteristics as compared to the calcined sample (Sample A). The hemolysis test demonstrates that the hydroxyapatite samples are highly hemocompatible biomaterial. Thus, the nanoporous hydroxyapatite could potentially be used in adsorption based biomedical applications.

\section{Acknowledgement}

The authors would like to thank Universiti Sains Malaysia, Ministry of Higher Education (MOHE) and Ministry of Science, Technology and Innovation (MOSTI) for their financial support.

\section{References}

1. V Sarath Chandra, G Baskar, R Suganthi, K Elayaraja, M Ahymah Joshy, et.al. (2012) Blood compatibility of iron-doped nanosize hydroxyapatite and its drug release. ACS Appl Mater Inter 4(3): 1200-1210.

2. W K Cheah, K Ishikawa, R Othman, F Y Yeoh (2017) Nanoporous biomaterials for uremic toxin adsorption in artificial kidney systems: A review. J Biomed Mater Res B 105(5): 1232-1240.

3. K Byrappa, T Adschiri (2007) Hydrothermal technology for nanotechnology. Prog Cryst Growth Ch 53: 117-166.

4. E Iyyappan, P Wilson, K Sheela, R Ramya (2016) Role of triton X-100 and hydrothermal treatment on the morphological features of nanoporous hydroxyapatite nanorods. Mat Sci Eng C-Mater 63: 554-562.

5. UTSeyfert, VBiehl, J Schenk (2002) In vitro hemocompatibility testing of biomaterials according to the ISO 10993-4. Biomol Eng 19(2-6): 91-96.

6. KS Lew, R Othman, FY Yeoh (2010) Synthesis and characterization of mesoporous hydroxyapatite,Advances in Science and Technology. Adv Sci Tech 63: 152-157.

7. S Jadalannagari, KDeshmukh, SRRamanan, MKowshik (2014) Antimicrobial activity of hemocompatible silver doped hydroxyapatite nanoparticles synthesized by modified sol-gel technique. Appl Nanoscience 4(2): 133-141.

8. SM Prabhu, S Meenakshi (2014) Synthesis of surface coated hydroxyapatite powders for fluoride removal from aqueous solution. Powder Technol 268(1): 306-315.

9. HY Juang, MH Hon (1996) Effect of calcination on sintering of hydroxyapatite. Biomaterials 17(21): 2059-2064.

10. G Radha, S Balakumar, B Venkatesan, E Vellaichamy (2015) Evaluation of hemocompatibility and in vitro immersion on microwave-assisted hydroxyapatite-alumina nanocomposites. Mater Sci Eng C-Mater 50: 143-150.

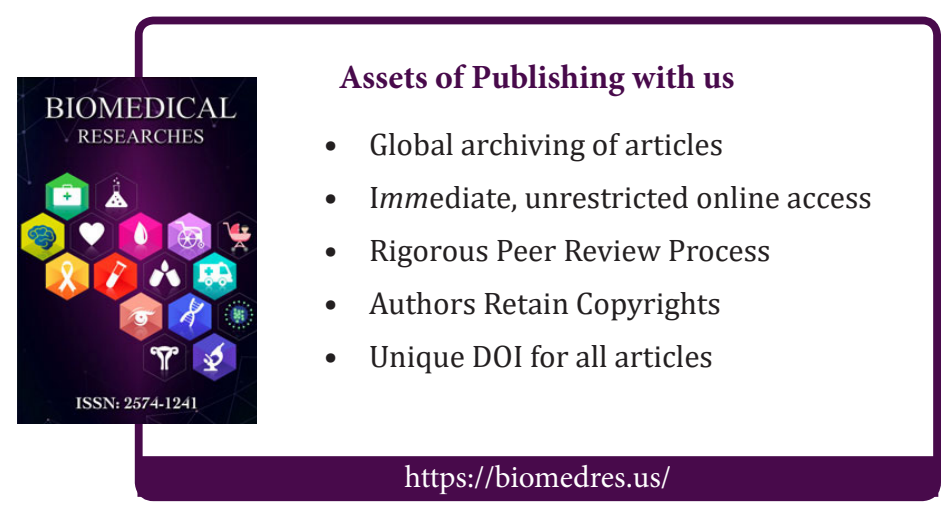

